\title{
Electrochemistry and Homogeneous Self-Exchange Kinetics of the Aqueous 12-Tungstoaluminate(5-/6-) \\ Couple
}

Almut Czap, Nancy I. Neuman and Thomas W. Swaddle*

Supporting Information 


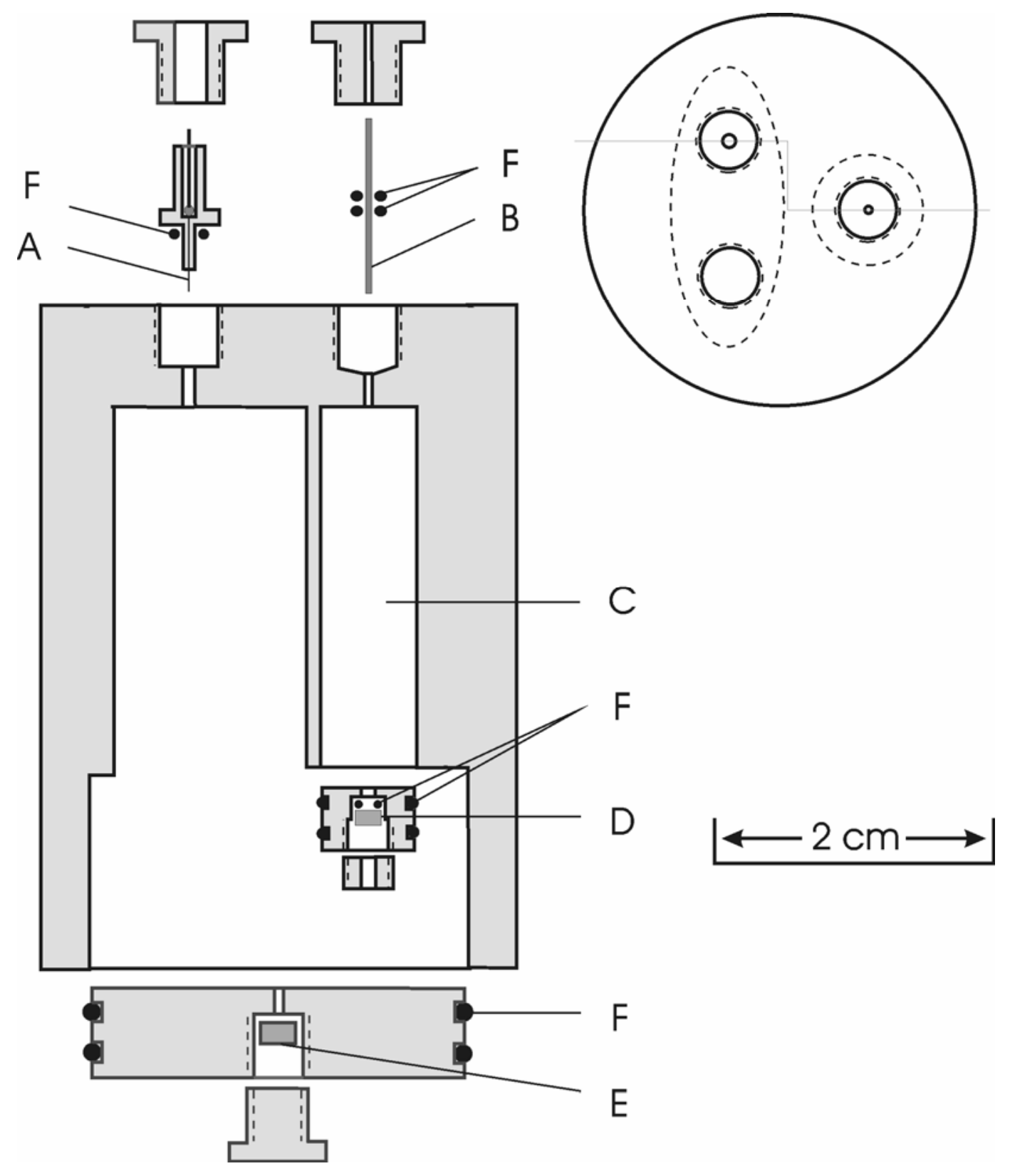

Figure S1. Electrochemical cell for high pressure service. A: working electrode. B: reference electrode. C: reference electrode compartment. D: Vycor frit. E: rubber disk seal. F: neoprene O-rings. Reprinted with permission from Neuman, N. I. M.Sc. Thesis, University of Calgary, 2005. Copyright 2005 Nancy I. Neuman. 


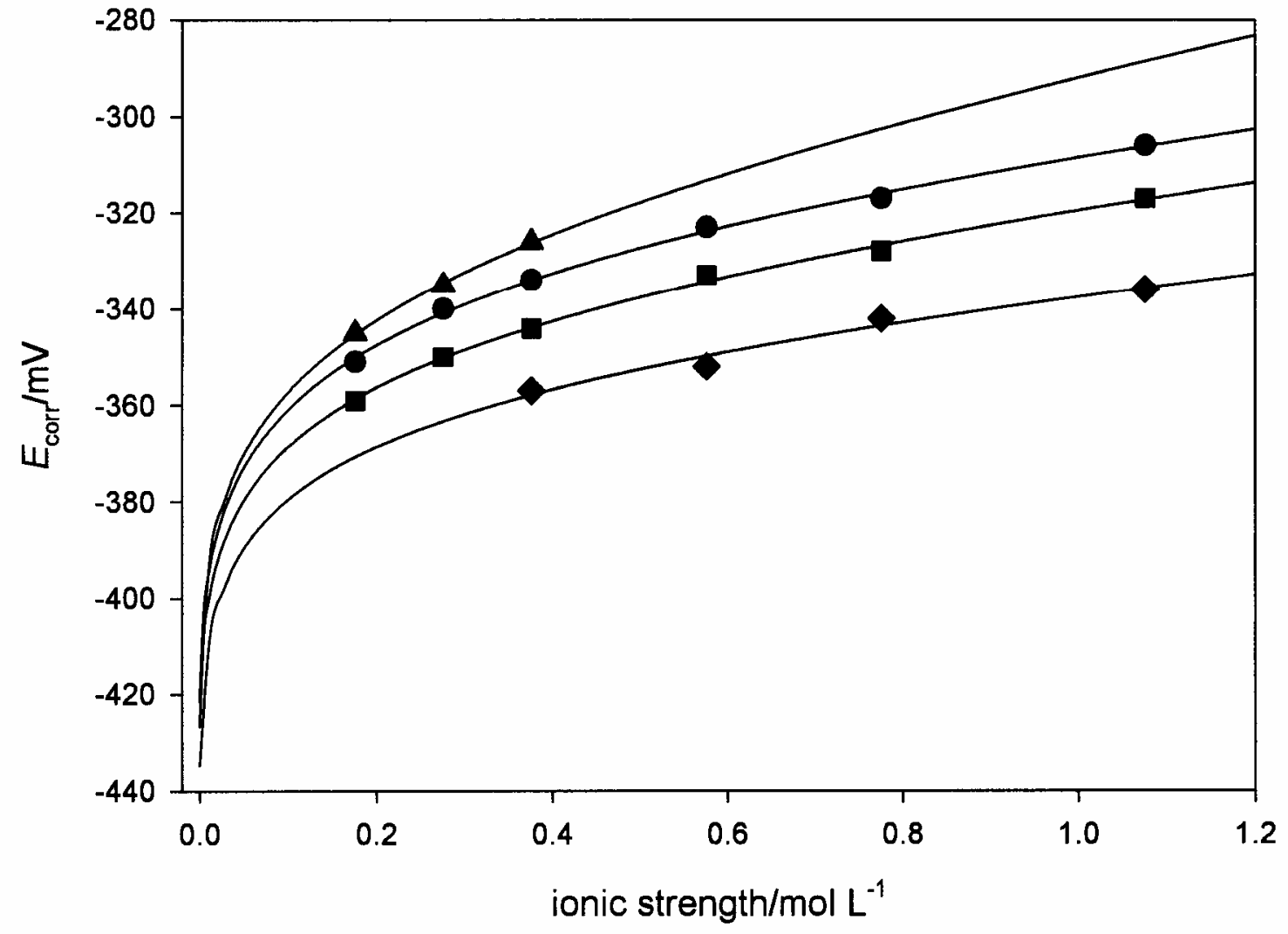

Figure S2 Dependence of liquid junction potential-corrected mid-point potentials $E_{\text {corr }}$ at $25 .{ }^{\circ} \mathrm{C}$ on ionic strength $I$ for supporting electrolytes $\mathrm{LiCl}(\bullet), \mathrm{NaCl}(\bullet), \mathrm{KCl}(\bullet), \mathrm{RbCl}$ ( $\mathbf{\Delta}$ ). Curves are least-squares fits of the $\mathrm{MCl}$ data to the extended Debye-Hückel equation $E_{\mathrm{m}}$ $=E_{\mathrm{m}}{ }^{0}+332.1 I^{1 / 2} /\left(1+3.286 a I^{1 / 2}\right)+c I$, with theoretical $a / \mathrm{nm}=0.918,0.836,0.748$ and 0.738 for $\mathrm{M}=\mathrm{Li}, \mathrm{Na}, \mathrm{K}$ and $\mathrm{Rb}$, respectively (see Table 2). 


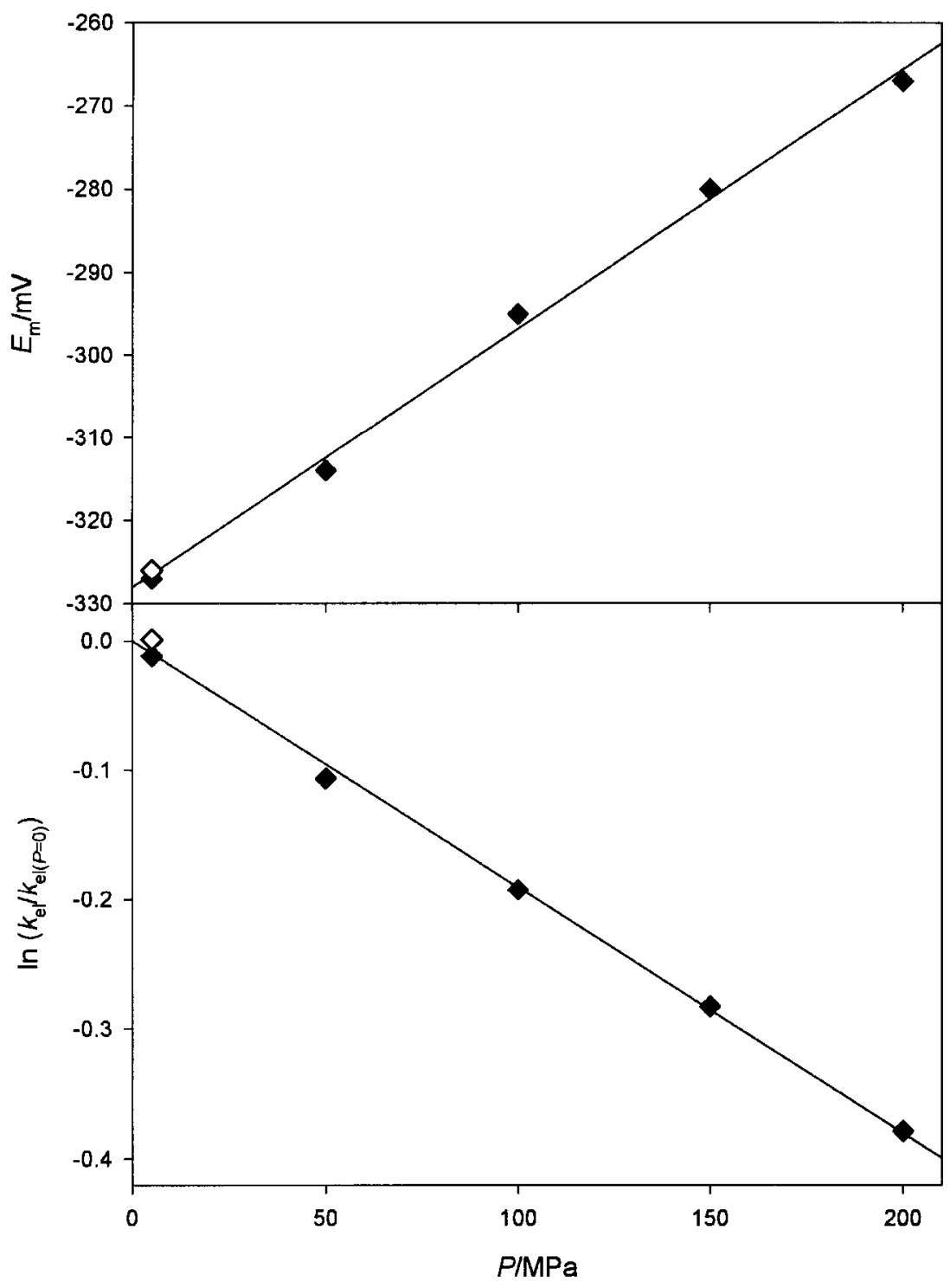

Figure S3. Pressure dependences of the CV mid-point potential $E_{\mathrm{m}}$ (upper frame) and the standard electrode reaction rate constant $k_{\mathrm{el}}$ (relative to zero applied pressure; lower frame) for $5.0 \mathrm{mmol} \mathrm{L}^{-1} \mathrm{AlW}_{12} \mathrm{O}_{40}{ }^{5-/ 6-}$ in $0.50 \mathrm{~mol} \mathrm{~L}^{-1} \mathrm{LiCl}$ at $25.0^{\circ} \mathrm{C}$. Counterion of the tungstoaluminate is $\mathrm{Li}^{+}$. Filled symbols: increasing pressure. Hollow symbols: decreasing pressure. 


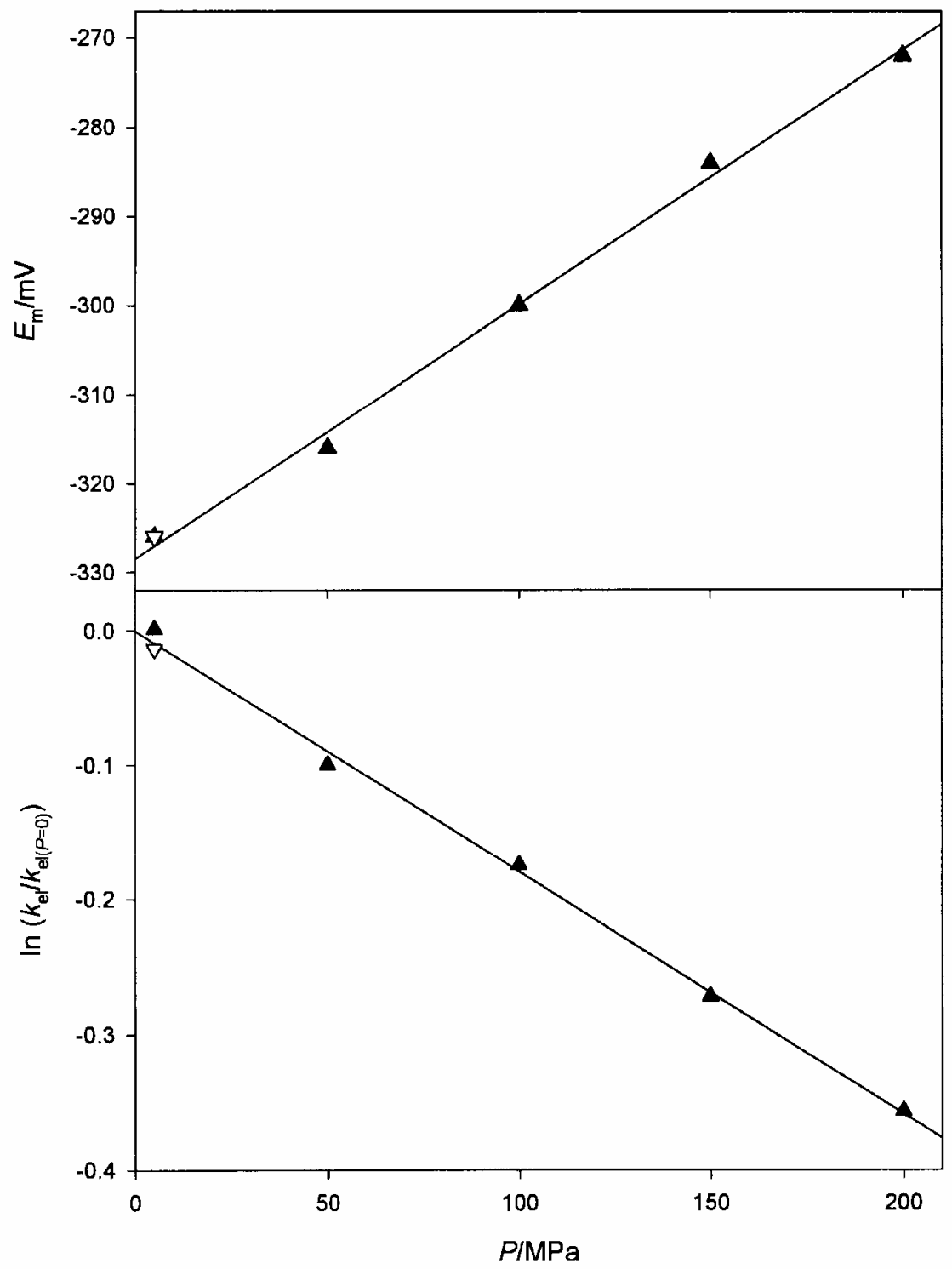

Figure S4. Pressure dependences of the CV mid-point potential $E_{\mathrm{m}}$ (upper frame) and the electrode reaction rate constant $k_{\mathrm{el}}$ (relative to zero applied pressure; lower frame) for 5.0 mmol L ${ }^{-1} \mathrm{AlW}_{12} \mathrm{O}_{40}{ }^{5-/ 6-}$ in $0.2 \mathrm{~mol} \mathrm{~L}^{-1} \mathrm{RbCl}$ at $25.0^{\circ} \mathrm{C}$. Counterion of the tungstoaluminate is $\mathrm{K}^{+}$. Filled symbols: increasing pressure. Hollow symbols: decreasing pressure. 


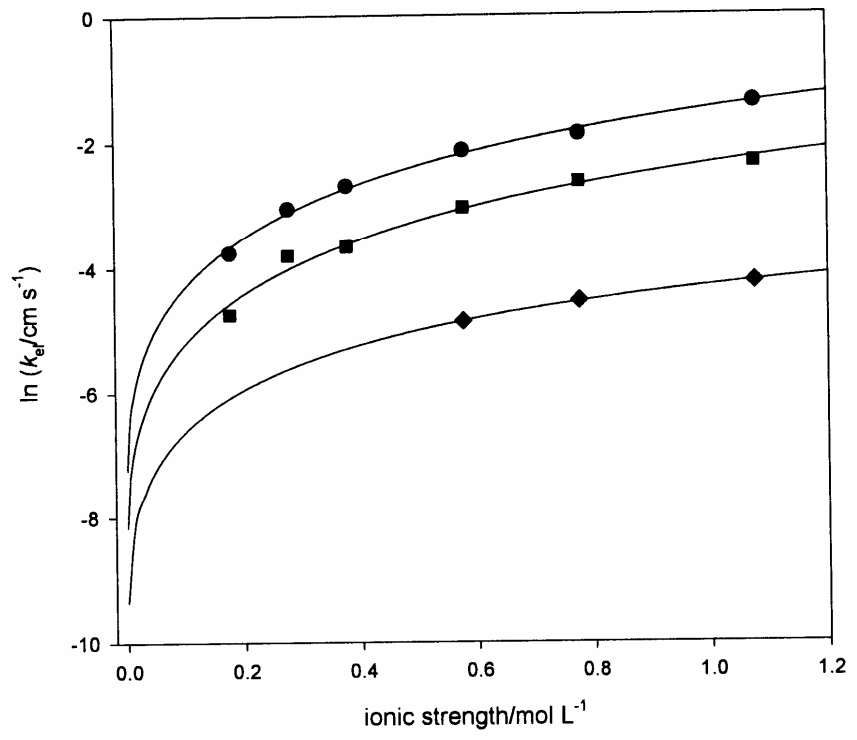

Figure S5. Dependence of $\mathrm{AlW}_{12} \mathrm{O}_{40}{ }^{5-/ 6-}$ electrode reaction rate constants at $25.0{ }^{\circ} \mathrm{C}$ on the ionic strength according to the Brønsted-Bjerrum-Christiansen equation modified for an electrode reaction (eq 9). Supporting electrolyte $\mathrm{LiCl}(\bullet), \mathrm{NaCl}(\boldsymbol{\bullet}), \mathrm{KCl}(\bullet)$.

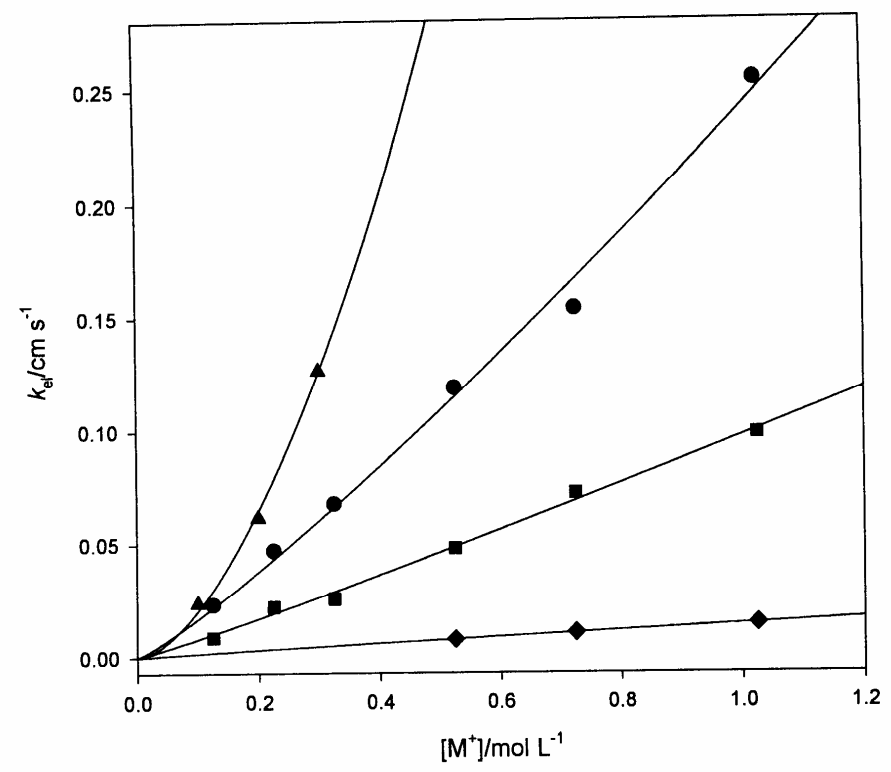

Figure S6. Empirical power equation $\left(k_{\mathrm{el}}=m\left[\mathrm{M}^{+}\right]^{p}\right.$, eq 10) fit of rate constants for the $\mathrm{AlW}_{12} \mathrm{O}_{40}{ }^{5-/ 6-}$ electrode reaction rate constants at $25.0^{\circ} \mathrm{C}$ to the cation concentration. 


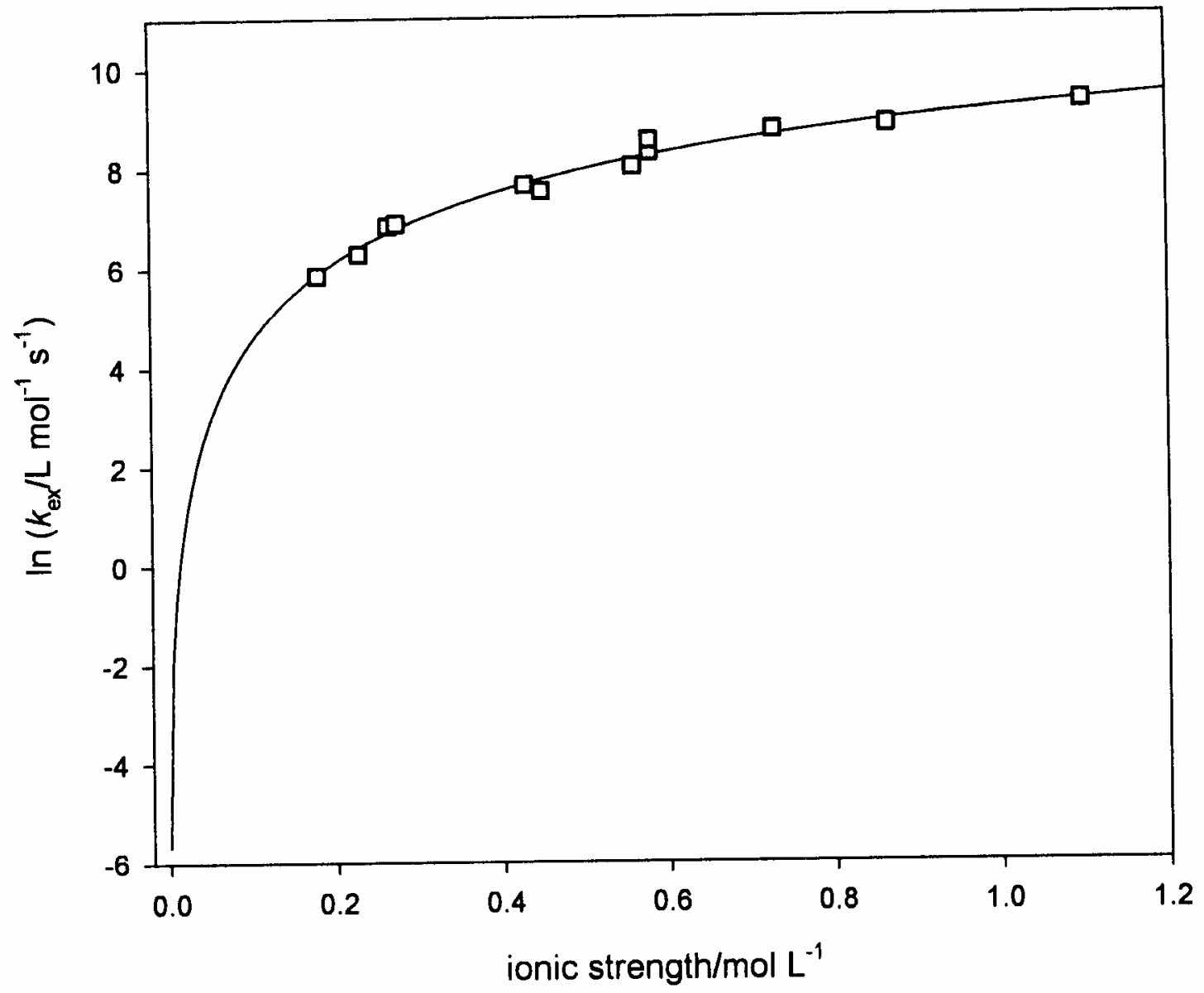

Figure S7. Non-linear least-squares fit of the rate constants of Geletii et al. (ref. 14) for the $\mathrm{AlW}_{12} \mathrm{O}_{40}{ }^{5-/ 6-}$ self-exchange reaction at $19.2{ }^{\circ} \mathrm{C}$ in $\mathrm{NaCl}$ media (phosphate buffer, $\mathrm{pH}$ 7.2) to ionic strength according to the Brønsted-Bjerrum-Christiansen equation (eq 8) with the ionic closest-approach distance $a$ as a free parameter. The fit gives $a=1.14 \pm 0.03 \mathrm{~nm}$ and $k_{\mathrm{ex}}{ }^{\mathrm{I}}=$ $0.0034 \pm 0.0012 \mathrm{~L} \mathrm{~mol}^{-1} \mathrm{~s}^{-1}$ with $r^{2}=0.9817$. 


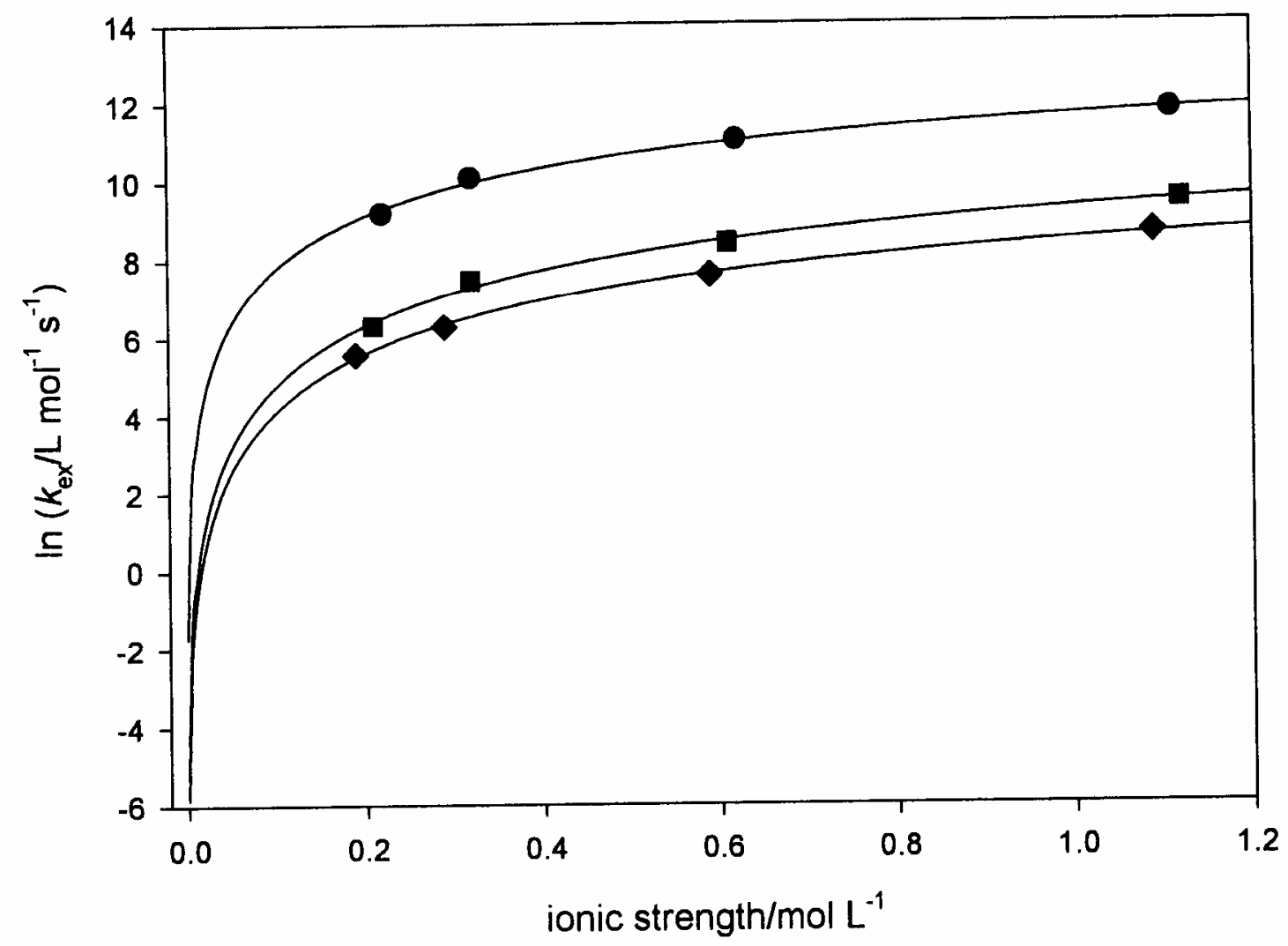

Figure S8. Nonlinear least-squares fit of $\mathrm{AlW}_{12} \mathrm{O}_{40}{ }^{5-/ 6-}$ self-exchange rate constants at 25.0 ${ }^{\circ} \mathrm{C}$ to the Brønsted-Bjerrum-Christiansen equation (eq 8) with the ionic closest-approach distance $a$ as a free parameter. Supporting electrolyte $\mathrm{LiCl}(\bullet), \mathrm{NaCl}(\bullet), \mathrm{KCl}(\bullet) ; a / \mathrm{nm}=$ $1.20 \pm 0.03,1.16 \pm 0.06,1.30 \pm 0.04 ; \ln \left(k_{\mathrm{ex}}{ }^{I=0} / \mathrm{L} \mathrm{mol}^{-1} \mathrm{~s}^{-1}\right)=-5.85 \pm 0.25,-5.34 \pm 0.47,-1.71$ $\pm 0.28 ; r^{2}=0.9971,0.9905,0.9943$, respectively. 
Table S1. Estimation of Liquid Junction Potentials $E_{\mathrm{lj}}{ }^{\mathrm{MCl}}$ for the $\mathrm{Ag} / \mathrm{AgCl} / \mathrm{NaCl}$ (aq, satd) Reference Electrode from Potentials $E^{\mathrm{MCl}}$ of $\mathrm{Ag} / \mathrm{AgCl} / \mathrm{MCl} / \mathrm{NaCl}(\mathrm{satd}) / \mathrm{AgCl} / \mathrm{Ag} \mathrm{Cells}{ }^{a}$

\begin{tabular}{|c|c|c|c|c|}
\hline $\mathrm{MCl}$ & {$[\mathrm{MCl}] / \mathrm{mol} \mathrm{L}^{-1}$} & $E^{\mathrm{MCl}} / \mathrm{mV}$ & 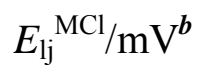 & $<E_{1 \mathrm{j}}^{\mathrm{MCl}}>/ \mathrm{mV}^{\mathrm{c}}$ \\
\hline \multirow[t]{6}{*}{$\mathrm{LiCl}$} & 0.10 & 89.1 & & -25.3 \\
\hline & 0.20 & 76.8 & & -21.5 \\
\hline & 0.30 & $69.5^{d}$ & & -19.6 \\
\hline & 0.50 & 60.5 & & -17.0 \\
\hline & 0.70 & $54.4^{d}$ & & -15.3 \\
\hline & 1.00 & 47.9 & & -13.6 \\
\hline \multirow[t]{6}{*}{$\mathrm{NaCl}$} & 0.10 & 88.0 & -24.2 & \\
\hline & 0.20 & 76.3 & -21.0 & \\
\hline & 0.30 & $68.9^{d}$ & -19.0 & \\
\hline & 0.50 & 60.1 & -16.6 & \\
\hline & 0.70 & $53.9^{d}$ & -14.8 & \\
\hline & 1.00 & 47.3 & -13.0 & \\
\hline \multirow[t]{6}{*}{$\mathrm{KCl}$} & 0.10 & 87.3 & & -23.5 \\
\hline & 0.20 & 74.4 & & -19.1 \\
\hline & 0.30 & $66.6^{d}$ & & -16.6 \\
\hline & 0.50 & 56.8 & & -13.3 \\
\hline & 0.70 & $50.5^{d}$ & & -11.4 \\
\hline & 1.00 & 43.7 & & -9.4 \\
\hline \multirow[t]{3}{*}{$\mathrm{RbCl}$} & 0.10 & 87.7 & & -23.9 \\
\hline & 0.20 & 74.7 & & -19.4 \\
\hline & 0.30 & 67.1 & & -17.2 \\
\hline
\end{tabular}

${ }^{a} 25.0{ }^{\circ} \mathrm{C} .{ }^{b} E_{\mathrm{lj}}{ }^{\mathrm{NaCl}}=\left(2 t_{+}-1\right) E^{\mathrm{NaCl}} / 2 t_{+}=-0.276 E^{\mathrm{NaCl}} .{ }^{c}$ Estimated by assuming $\left(E^{\mathrm{MCl}}-E^{\mathrm{NaCl}}\right)$ is due solely to difference in liquid junction potentials; thus, $<E_{\mathrm{lj}}^{\mathrm{MCl}}>=E_{\mathrm{lj}}^{\mathrm{NaCl}}-\left(E^{\mathrm{MCl}}-E^{\mathrm{NaCl}}\right)$. ${ }^{d}$ Interpolated using linear dependence of $E^{\mathrm{MCl}}$ on $\log [\mathrm{MCl}]$. 
Table S2. Theoretical (Fuoss) Ion Pair Formation Constants $K_{\mathrm{IP}}$ for $\mathrm{AlW}_{12} \mathrm{O}_{40}{ }^{5-}$ and $\mathrm{AlW}_{12} \mathrm{O}_{40}{ }^{6-}$ with Alkali Metal Ions, and Calculated Effect $\Delta E_{\mathrm{IP}}$ of Ion Pairing on the $\mathrm{AlW}_{12} \mathrm{O}_{40}{ }^{5-/ 6-}$ Electrode Potential. $^{a}$

\begin{tabular}{|c|c|c|c|c|c|c|c|c|}
\hline & \multirow{2}{*}{ cation } & \multicolumn{7}{|c|}{ ionic strength $/ \mathrm{mol} \mathrm{L}^{-1}$} \\
\hline & & 0.0 & 0.176 & 0.276 & 0.376 & 0.576 & 0.776 & 1.076 \\
\hline \multirow{4}{*}{$K_{\mathrm{IP}}{ }^{5-}\left(\mathrm{AlW}_{12} \mathrm{O}_{40}{ }^{5-}\right)$} & $\mathrm{Li}^{+}$ & 96 & 10.9 & 8.8 & 7.7 & 6.4 & 5.7 & 5.0 \\
\hline & $\mathrm{Na}^{+}$ & 106 & 10.8 & 8.5 & 7.3 & 5.9 & 5.2 & 4.5 \\
\hline & $\mathrm{K}^{+}$ & 126 & 11.2 & 8.5 & 7.1 & 5.6 & 4.8 & 4.1 \\
\hline & $\mathrm{Rb}^{+}$ & 129 & 11.2 & 8.6 & 7.1 & 5.6 & 4.8 & 4.0 \\
\hline \multirow[t]{4}{*}{$K_{\mathrm{IP}}{ }^{6-}\left(\mathrm{AlW}_{12} \mathrm{O}_{40}{ }^{6-}\right)$} & $\mathrm{Li}^{+}$ & 209 & 15.4 & 11.9 & 10.1 & 8.1 & 7.0 & 6.1 \\
\hline & $\mathrm{Na}^{+}$ & 250 & 16.1 & 12.1 & 10.0 & 7.8 & 6.6 & 5.6 \\
\hline & $\mathrm{K}^{+}$ & 328 & 17.9 & 13.0 & 10.4 & 7.8 & 6.5 & 5.3 \\
\hline & $\mathrm{Rb}^{+}$ & 340 & 18.2 & 13.1 & 10.5 & 7.9 & 6.5 & 5.3 \\
\hline \multirow[t]{4}{*}{$\Delta E_{\mathrm{IP}} / \mathrm{mV}$} & $\mathrm{Li}^{+}$ & 0.0 & 5.0 & 5.2 & 5.1 & 4.8 & 4.4 & 4.1 \\
\hline & $\mathrm{Na}^{+}$ & 0.0 & 5.8 & 6.0 & 5.9 & 5.5 & 5.2 & 4.8 \\
\hline & $\mathrm{K}^{+}$ & 0.0 & 7.1 & 7.3 & 7.1 & 6.6 & 6.2 & 5.7 \\
\hline & $\mathrm{Rb}^{+}$ & 0.0 & 7.3 & 7.4 & 7.2 & 6.7 & 6.3 & 5.8 \\
\hline
\end{tabular}

${ }^{a}$ For water at $25.0{ }^{\circ} \mathrm{C}$; units of $K_{\mathrm{IP}}$ are $\mathrm{L} \mathrm{mol}^{-1}$; ionic strength effect calculated from eq 1 , with $a / \mathrm{nm}=0.918,0.836,0.748$ and 0.738 for $\mathrm{M}=\mathrm{Li}, \mathrm{Na}, \mathrm{K}$ and $\mathrm{Rb}$, respectively (see Table 2). 
Table S3. Effect of Temperature on the Mean Diffusion Coefficient of $\mathrm{AlW}_{12} \mathrm{O}_{40}{ }^{5-/ 6-}$ in Aqueous Solution ${ }^{a, b}$

\begin{tabular}{llllll}
\hline$T /{ }^{\circ} \mathrm{C}$ & 20.0 & 25.0 & 30.0 & 35.0 & 40.0 \\
\hline$\eta / \mathrm{mPa} \mathrm{s}^{a}$ & 1.002 & 0.890 & 0.798 & 0.720 & 0.653 \\
$D / 10^{-6} \mathrm{~cm}^{2} \mathrm{~s}^{-1 b}$ & 3.8 & 4.4 & 5.0 & 5.6 & 6.3
\end{tabular}

${ }^{a}$ Sengers, J. V.; Kamgar-Parsi, B. J. Phys. Chem. Ref. Data 1984, 13, 185.

${ }^{b}$ Calculated from $D=4.4 \times 10^{-6} \mathrm{~cm}^{2} \mathrm{~s}^{-1}$ at $25.0^{\circ} \mathrm{C}$ (see text). 
Table S4. Electrolyte, Temperature ${ }^{a}$ and Pressure ${ }^{b}$ Dependences of the Transfer Coefficient $\alpha$ for the Aqueous $\mathrm{AlW}_{12} \mathrm{O}_{40}{ }^{5-/ 6-} \mathrm{Couple}$ at a Gold Wire Electrode.

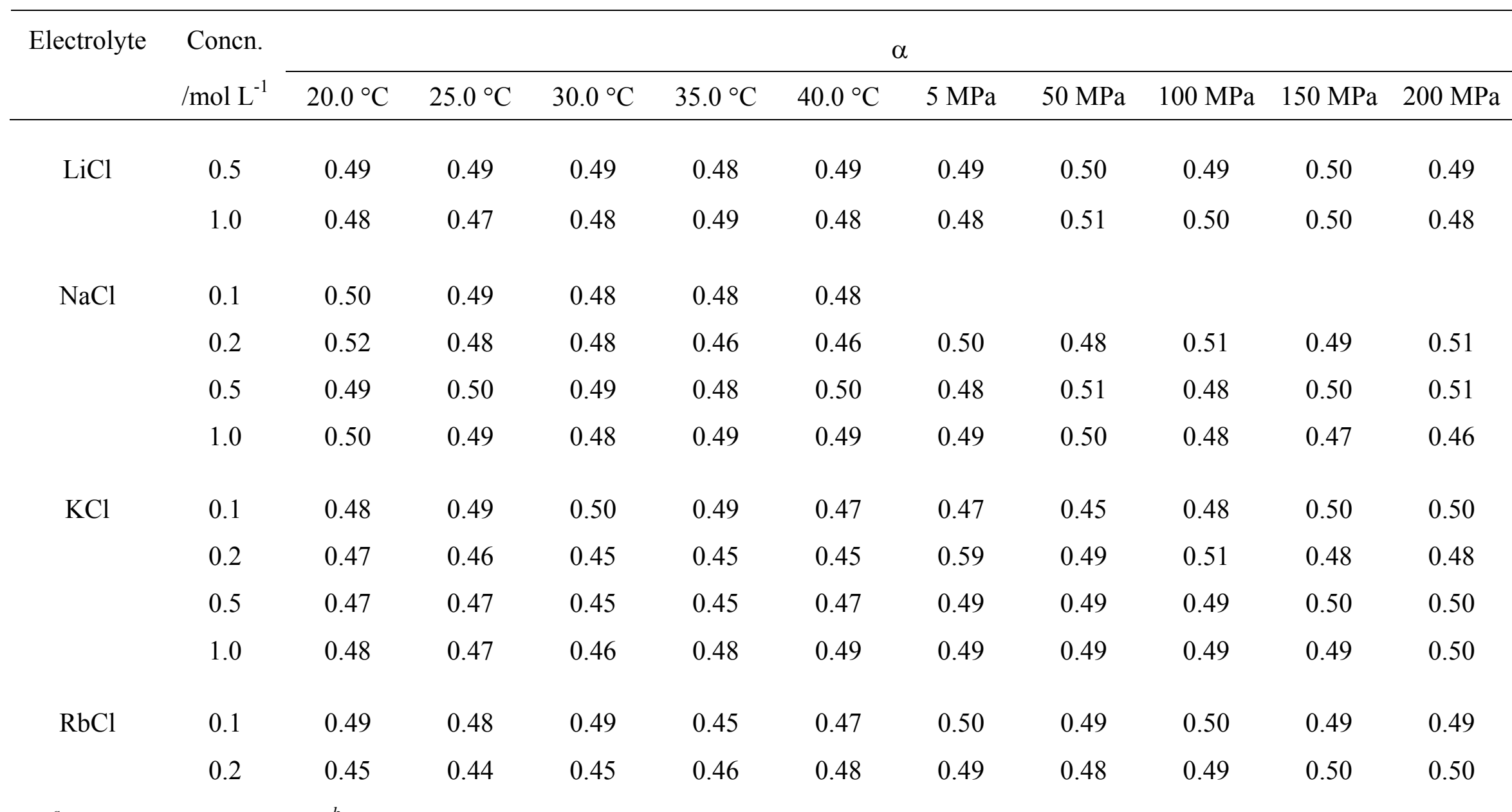

${ }^{a}$ In glass cell at $0.1 \mathrm{MPa} .{ }^{b}$ In high pressure cell at $25.0^{\circ} \mathrm{C}$. 
Table S5. Electrolyte, Temperature, ${ }^{a}$ and Pressure ${ }^{b}$ Dependences of the Uncompensated Resistance.

\begin{tabular}{|c|c|c|c|c|c|c|c|c|c|c|c|}
\hline \multirow[t]{2}{*}{ Electrolyte } & \multirow{2}{*}{$\begin{array}{l}\text { Concn. } \\
/ \mathrm{mol} \mathrm{L}^{-1}\end{array}$} & \multicolumn{10}{|c|}{ Uncompensated Resistance $R_{\mathrm{u}} / \Omega$} \\
\hline & & $20.0^{\circ} \mathrm{C}$ & $25.0^{\circ} \mathrm{C}$ & $30.0^{\circ} \mathrm{C}$ & $35.0^{\circ} \mathrm{C}$ & $40.0^{\circ} \mathrm{C}$ & $5 \mathrm{MPa}$ & $50 \mathrm{MPa}$ & $100 \mathrm{MPa}$ & $150 \mathrm{MPa}$ & $200 \mathrm{MPa}$ \\
\hline \multirow[t]{2}{*}{$\mathrm{LiCl}$} & 0.5 & 68 & 59 & 54 & 49 & 49 & 118 & 109 & 106 & 110 & 99 \\
\hline & 1.0 & 28 & 26 & 24 & 23 & 21 & 56 & 53 & 55 & 50 & 51 \\
\hline \multirow[t]{4}{*}{$\mathrm{NaCl}$} & 0.1 & 139 & 128 & 119 & 119 & 119 & & & & & \\
\hline & 0.2 & 95 & 85 & 75 & 67 & 63 & 126 & 119 & 115 & 113 & 111 \\
\hline & 0.5 & 48 & 47 & 40 & 36 & 34 & 60 & 59 & 57 & 56 & 55 \\
\hline & 1.0 & 24 & 22 & 19 & 18 & 16 & 38 & 35 & 34 & 34 & 33 \\
\hline \multirow[t]{4}{*}{$\mathrm{KCl}$} & 0.1 & 153 & 138 & 123 & 114 & 113 & 213 & 199 & 195 & 194 & 194 \\
\hline & 0.2 & 92 & 79 & 71 & 65 & 59 & 129 & 126 & 123 & 123 & 118 \\
\hline & 0.5 & 38 & 35 & 31 & 29 & 27 & 66 & 59 & 58 & 55 & 57 \\
\hline & 1.0 & 19 & 17 & 17 & 15 & 14 & 34 & 32 & 29 & 29 & 28 \\
\hline \multirow[t]{2}{*}{$\mathrm{RbCl}$} & 0.1 & 169 & 151 & 140 & 123 & 114 & 260 & 264 & 257 & 251 & 258 \\
\hline & 0.2 & 97 & 86 & 78 & 71 & 68 & 179 & 178 & 160 & 156 & 158 \\
\hline
\end{tabular}

${ }^{a}$ In glass cell at $0.1 \mathrm{MPa} .{ }^{b}$ In high pressure cell at $25.0^{\circ} \mathrm{C}$. 
Table S6. Parameters Representing the Temperature Dependences of the Electrode Reaction Rate Constant of the Aqueous $\mathrm{AlW}_{12} \mathrm{O}_{40}{ }^{5-/ 6-}$ Couple at a Gold Electrode.

\begin{tabular}{|c|c|c|c|c|c|c|c|c|}
\hline $\mathrm{MCl}$ & & \multicolumn{5}{|c|}{$k_{\mathrm{el}} / 10^{-2} \mathrm{~cm} \mathrm{~s}^{-1}\left(\right.$ temp $\left./{ }^{\circ} \mathrm{C}\right)$} & \multirow{2}{*}{$\begin{array}{c}\Delta H_{\mathrm{el}}^{\ddagger} \\
/ \mathrm{kJ} \mathrm{mol}^{-1} \\
34.1 \pm 0.5\end{array}$} & \multirow{2}{*}{$\begin{array}{c}\Delta S^{\ddagger} \\
/ \mathrm{J} \mathrm{K}^{-1} \mathrm{~mol}^{-1} \\
-170.8 \pm 1.7\end{array}$} \\
\hline \multirow[t]{2}{*}{$\mathrm{LiCl}^{a}$} & 0.5 & $0.60(20.1)$ & $0.77(25.05)$ & $0.96(30.1)$ & $1.25(35.05)$ & $1.56(39.95)$ & & \\
\hline & 1.0 & $1.06(19.95)$ & $1.45(24.95)$ & $1.83(30.0)$ & $2.34(34.9)$ & $2.91(39.9)$ & $35.8 \pm 1.0$ & $-160.1 \pm 3.4$ \\
\hline \multirow[t]{4}{*}{$\mathrm{NaCl}^{a}$} & 0.1 & $0.73(20.1)$ & $0.87(25.0)$ & $1.13(30.2)$ & $1.41(35.05)$ & $1.84(40.4)$ & $32.8 \pm 1.4$ & $-174.1 \pm 4.5$ \\
\hline & 0.2 & $1.89(20.25)$ & $2.23(25.0)$ & $2.79(30.0)$ & $3.60(35.05)$ & $4.49(40.1)$ & $31.4 \pm 1.4$ & $-171.0 \pm 4.6$ \\
\hline & 0.5 & $3.83(20.1)$ & $4.79(25.15)$ & $6.01(30.3)$ & $7.69(35.0)$ & $9.21(40.3)$ & $31.3 \pm 1.0$ & $-165.1 \pm 3.3$ \\
\hline & 1.0 & 7.91 (19.95) & $9.85(25.0)$ & $12.5(29.95)$ & $15.8(35.05)$ & $19.1(40.2)$ & $31.3 \pm 0.7$ & $-158.9 \pm 2.3$ \\
\hline \multirow[t]{4}{*}{$\mathrm{KCl}^{a}$} & 0.1 & $1.99(20.5)$ & $2.34(25.2)$ & $2.85(30.8)$ & $3.29(35.2)$ & $3.93(40.0)$ & $24.0 \pm 0.4$ & $-195.4 \pm 1.5$ \\
\hline & 0.2 & $3.47(19.9)$ & $4.03(25.4)$ & $4.75(30.6)$ & $5.62(35.6)$ & $6.83(40.3)$ & $23.3 \pm 1.3$ & $-192.9 \pm 4.4$ \\
\hline & 0.5 & $10.0(19.9)$ & $12.2(25.0)$ & $14.6(30.4)$ & $16.8(35.4)$ & $20.4(40.1)$ & $23.1 \pm 0.9$ & $-185.2 \pm 3.1$ \\
\hline & 1.0 & $20.4(20.2)$ & $25.1(25.3)$ & $29.1(30.7)$ & $32.4(35.2)$ & $38.9(40.1)$ & $21.5 \pm 1.3$ & $-182.4 \pm 3.5$ \\
\hline \multirow[t]{2}{*}{$\mathrm{RbCl}^{b}$} & 0.1 & $2.36(20.1)$ & $2.70(25.4)$ & $3.17(30.1)$ & $3.65(35.1)$ & $4.02(40.15)$ & $18.5 \pm 0.9$ & $-213.0 \pm 3.0$ \\
\hline & 0.2 & $5.93(20.15)$ & $6.47(25.15)$ & $7.40(30.1)$ & $8.53(34.9)$ & $9.74(39.95)$ & $17.1 \pm 1.1$ & $-210.3 \pm 3.7$ \\
\hline
\end{tabular}


Table S7. Temperature Dependence of Rate Constants $k_{\mathrm{ex}} / 10^{3} \mathrm{~L} \mathrm{~mol}^{-1} \mathrm{~s}^{-1}$ for Homogeneous Self-Exchange of the Aqueous $\mathrm{AlW}_{12} \mathrm{O}_{40}{ }^{5-/ 6-}$ Couple

\begin{tabular}{|c|c|c|c|c|c|c|}
\hline \multirow[t]{2}{*}{$\mathrm{MCl}$} & \multirow{2}{*}{$\begin{array}{c}{\left[\mathrm{M}^{+}\right]} \\
/ \mathrm{mol} \mathrm{L}^{-1}\end{array}$} & \multicolumn{5}{|c|}{ Temperature $/{ }^{\circ} \mathrm{C}$} \\
\hline & & 10.0 & 20.0 & 25.0 & 30.0 & 40.0 \\
\hline $\mathrm{LiCl}$ & 0.125 & $0.21 \pm 0.01$ & $0.24 \pm 0.01$ & $0.26 \pm 0.01$ & $0.28 \pm 0.01$ & $0.33 \pm 0.01$ \\
\hline $\mathrm{LiCl}$ & 0.225 & $0.41 \pm 0.01$ & $0.50 \pm 0.02$ & $0.53 \pm 0.07$ & $0.60 \pm 0.02$ & $0.71 \pm 0.01$ \\
\hline $\mathrm{LiCl}$ & 0.525 & $1.43 \pm 0.05$ & $1.8 \pm 0.1$ & $1.99 \pm 0.08$ & $2.3 \pm 0.2$ & $3.1 \pm 0.1$ \\
\hline $\mathrm{LiCl}$ & 1.025 & $4.17 \pm 0.01$ & $4.9 \pm 0.4$ & $5.67 \pm 0.06$ & $6.39 \pm 0.06$ & $7.54 \pm 0.04$ \\
\hline $\mathrm{NaCl}$ & 0.125 & $0.48 \pm 0.03$ & $0.53 \pm 0.08$ & $0.55 \pm 0.01$ & $0.57 \pm 0.04$ & $0.63 \pm 0.02$ \\
\hline $\mathrm{NaCl}$ & 0.225 & $1.40 \pm 0.02$ & $1.59 \pm 0.01$ & $1.7 \pm 0.1$ & $1.85 \pm 0.03$ & $2.17 \pm 0.01$ \\
\hline $\mathrm{NaCl}$ & 0.525 & $3.78 \pm 0.08$ & $4.23 \pm 0.08$ & $4.5 \pm 0.1$ & $4.67 \pm 0.01$ & $5.01 \pm 0.07$ \\
\hline $\mathrm{NaCl}$ & 1.025 & $11.14 \pm 0.09$ & $12.30 \pm 0.1$ & $12.89 \pm 0.02$ & $13.8 \pm 0.5$ & $15.3 \pm 0.4$ \\
\hline $\mathrm{KCl}$ & 0.125 & $10.2 \pm 0.4$ & $10.0 \pm 0.8$ & $9.8 \pm 0.1$ & $9.8 \pm 0.3$ & $9.6 \pm 0.3$ \\
\hline $\mathrm{KCl}$ & 0.225 & $25.4 \pm 0.9$ & $24.3 \pm 0.1$ & $24.0 \pm 0.2$ & $23.2 \pm 0.1$ & $22.7 \pm 0.1$ \\
\hline $\mathrm{KCl}^{a}$ & 0.525 & $68 \pm 2$ & $62.3 \pm 0.8$ & $62 \pm 3$ & $59.5 \pm 0.7$ & $55.3 \pm 0.2$ \\
\hline $\mathrm{KCl}^{a}$ & 0.525 & $68 \pm 2$ & $62 \pm 3$ & $61.6 \pm 0.8$ & $59.7 \pm 0.4$ & $57.6 \pm 0.1$ \\
\hline $\mathrm{KCl}^{b}$ & 1.025 & 129.6 & 129.2 & 128.6 & 128.4 & 127.6 \\
\hline $\mathrm{KCl}^{b, c}$ & 1.025 & $131 \pm 3$ & $130 \pm 3$ & $129 \pm 3$ & $130 \pm 3$ & $128 \pm 3$ \\
\hline $\mathrm{RbCl}^{d}$ & 0.100 & $62 \pm 4$ & $55 \pm 5$ & $51.8 \pm 0.1$ & $46 \pm 5$ & $41.2 \pm 0.8$ \\
\hline $\mathrm{RbCl}^{a, d}$ & 0.200 & $129 \pm 4$ & $126 \pm 4$ & $124 \pm 5$ & $117 \pm 8$ & $115 \pm 5$ \\
\hline $\mathrm{Rb}_{2} \mathrm{CO}_{3}{ }^{a, d}$ & 0.200 & $127 \pm 1$ & $125 \pm 5$ & $122 \pm 5$ & $116 \pm 7$ & $113 \pm 4$ \\
\hline $\mathrm{KCF}_{3} \mathrm{SO}_{3}$ & 0.525 & $60.3 \pm 4.5$ & $54.3 \pm 4.4$ & $54.4 \pm 5.1$ & $52.4 \pm 4.9$ & $57 \pm 7^{e}$ \\
\hline
\end{tabular}

${ }^{a} k_{\text {ex }}$ obtained from the slow-exchange calculation using deconvoluted line widths at half maximum. ${ }^{b} k_{\mathrm{ex}}$ obtained from the intermediate-exchange equation (equation 5.2); only one $k_{\mathrm{ex}}$ value. ${ }^{c} 2.5 \mathrm{mmol} \mathrm{L}^{-1}$ total $\mathrm{AlW}_{12} \mathrm{O}_{40}{ }^{5-/ 6-}$ concentration. ${ }^{d} 0.025 \mathrm{~mol} \mathrm{~L}^{-1} \mathrm{Li}^{+}$present (from $\left.\mathrm{Li}_{5} \mathrm{AlW}_{12} \mathrm{O}_{40}\right)$. ${ }^{e}$ Slight precipitate visible - not included in Eyring fit. 
Table S8. Concentration Dependence of Rate Constants $k_{\mathrm{ex}}$ for Homogeneous Self-Exchange of the Aqueous $\mathrm{AlW}_{12} \mathrm{O}_{40}{ }^{5-/ 6-}$ Couple $^{a}$

\begin{tabular}{|c|c|c|c|c|}
\hline $\begin{array}{c}{\left[\mathrm{AlW}_{12} \mathrm{O}_{40}^{5-}\right]} \\
/ \mathrm{mmol} \mathrm{L}^{-1}\end{array}$ & $\begin{array}{c}{\left[\mathrm{AlW}_{12} \mathrm{O}_{40}{ }^{6-}\right]} \\
/ \mathrm{mmol} \mathrm{L}^{-1}\end{array}$ & $\begin{array}{c}k_{\mathrm{ex}}{ }^{\mathrm{d}} \\
/ 10^{3} \mathrm{~L} \mathrm{~mol}^{-1} \mathrm{~s}^{-1}\end{array}$ & $\begin{array}{c}k_{\mathrm{ex}}^{\mathrm{p}} \\
/ 10^{3} \mathrm{~L} \mathrm{~mol}^{-1} \mathrm{~s}^{-1}\end{array}$ & $\begin{array}{l}\text { Average }=k_{\mathrm{ex}} \\
/ 10^{3} \mathrm{~L} \mathrm{~mol}^{-1} \mathrm{~s}^{-1}\end{array}$ \\
\hline 3.63 & 1.37 & 6.1 & 5.4 & 5.8 \\
\hline 2.65 & 2.35 & 5.6 & 5.5 & 5.6 \\
\hline 1.59 & 3.41 & 5.2 & 6.0 & 5.6 \\
\hline
\end{tabular}

${ }^{a}$ Counterion $\mathrm{Na}^{+}, 0.5 \mathrm{~mol} \mathrm{~L}^{-1} \mathrm{NaCl}, 1.0 \mathrm{mmol} \mathrm{L}{ }^{-1} \mathrm{HClO}_{4}, 25.0{ }^{\circ} \mathrm{C} ; k_{\mathrm{ex}}{ }^{\mathrm{d}}$ from ${ }^{27} \mathrm{Al}$ line widths of diamagnetic $\mathrm{AlW}_{12} \mathrm{O}_{40}{ }^{5-}, k_{\mathrm{ex}}{ }^{\mathrm{p}}$ from ${ }^{27} \mathrm{Al}$ line widths of paramagnetic $\mathrm{AlW}_{12} \mathrm{O}_{40}{ }^{6-}$. 